der Erde, wobei ihn hauptsächlich das Steingewolbo geschützt haben mag, erhalten kann, so dafs cr nech einem Zeitraumo ron 30 Jahren noch dieselben Dienste leistet.

\title{
Dritie Abtheilung.
}

iis is llen.

20

Ueber metallene Schomsteine and die Nachtheile ihrer Anwcrdung; von F. Kuhimanm,

(Ausugg der vain Verf. mitgetheilten Abhandiang.)

Der Verf, der Mitglied eine: zur Untarsuch wng les nachtheiligen Einfluswes kupferner whi eiserser solursteteine autgestellten Commiswion war, machte bei dieser Gelcgenheit, zu Houbaix und anderen Stälten in Frankreich, wo solche Schornsteine in nicht unbeträchtlicher $\mathrm{Zah}$ sich vorfinden, folgende wichtige Beobachtungen:

Fis fiel in einen Schorustein, un den unmittelber eia anderer, der durch ein kupfernes Kohr vertängert war, angebaut war, ein Ihufs herab, der betrïhdiche Blengen eines lïslichen Kupfergalxea enthielt. Die Ursache der Kratugung dieaes kupferhaltigen Rufses liegt in der als Brennmaterial angewendeter Steinkolsle. Dio Steiuhoble enthäit wechselude Mengeh von Schwefelhiesen, Doppelt.-Scliwafeloisen, welches bei hoher Temperaiur sich in einfach Schwefoleisen amwandelt, 
indem es die Hälfte seines Scluw cfels abgibt. Durch fortgesetzte und gut regulirte Speisung des Heerdes verbrennt dieser Schwefel zu schwefliger Säure, allein in dem Augenblick des Aufgebens, wenn der Heerd eine grofse Quantităt Brennmaterial enthält, ist der durch den Rost sireichende Sauerstoff nicht hinreichend oder die Temperatur ist zu sehr erniedrigt, um die fintäudung der Gasarten zu bewirken, und ein Theil des Schwefels entweicht als Scliwefelwasserstoff oder Schwefelwasserstoff-Ammoniak, weleher beim Durchstreichen doo kupfernen Rohrs einen Theil dieses Metalls in Sulfür verwaudelt.

Die Umwandlung des Kupfersulfiirs in Sulphat erklärt sich leicht durch den Einfufs der Luft, wekche wëhrend der Nacht durch das Kamin atreicht; diese Unwandlung ist bemerkenswerth noch defslalb, weil das erzeugte Sulphat krystallinisch ist. Diese! Kupferagiz wird zum Theil durch den Luftstron aus dem Schornstein mit fortgerissen, und war in dem in Cisternen aufgefungenen, ron dem Dache des Hausen ahgeflossenen Regenwasser nachweisbar, so dus es unter ungünstigen Umständen wohl in soleher Quantität darin vorhanden seyn konste, un einen nachtheiligen Wintuls auf die Per. sonen auszü̈ben, die ron diesem Wasser Gebrauch machten. $\Lambda$ ufser diesen Nachtheilen bringen aber die netallenen Sehornwteinc noch eine andere Gefalır mit sich, nümlich die des Brandes, durch Aussprühung einer betrüchtlichen Zahl von Funken, welche selbet weit davon entlegene Wohaungen erreichen, wonn eine Entzündang in dem Sehornstein anobricht.

Nu: seltene Beispiele bestätigen die Möglichkeit der Entzündung der flufisablagerungen an den Wanden von Schornsteinen von Dampfmaschinen, wenn sie aus Mnuerwerk bestehen, während die Erfahrong gezeigt hat, dals diese Fntzündungen sehr lüufg in Srhornsteinen sind, die durch ein kupfernes llohr verlangert sind. Dis Verengerung, welche die 
Oefriung des Schornstcins beim Eintritt in das Kupferrohr erleidet, kann dorch Verlangsamung des Zugs in dem unteren Theile, die bildung aines reichlichen $R$ ufsabsaizes an den gemeneqten Wändess veranlasset, aber es gib zuch noch einen modern Grund, der at Knizuading der mit kupfernen Röhren verehenen Schornsteine bejtragen sann. Der Hufs, der sich Ieicht an die Meisllwand anlegt, kann durch Condensation des Sayerstoffs des luft die frehiwillige Entzündung bedingen, sclist wenn die von Heerde augheliende Wärne weniz hetriblillch ist, ex kann endich die angufiblute Umwandiung des Kupfersulfirs in suiphat bisweilen die bedingende Ersache dieser Entzinuiungen seyn orler k cnigsteus mächtig dazo beitragen durch Erzengug einer hinteiclienden Temperaturer" höhtung in mitlen einer so entzündlichen und so weaig die Wärine leitenden Masse dss der hufs. In diesen letateren Falle wirde di. Wirtung einigermaisen mit ber des GayLussac'schen Pyrophurs analog seyn. In Roubaix, wo es

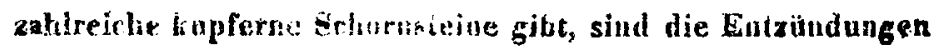
ziemlich häutig, dis in ter ivacht, fir die Vachbarn oft ein Gegenstand der Henumbigung sind. Ber Brand brach sehr oft aus, lange mathitern dus Feuer auf dem Ifeerde gelöscht und selbst weun die 7 age geschlossen waren.

In einem Schornsteine zis Lille, dessen Mauerwark 26 Meter hoch end dureh bin \& Meter langes Kupferrohr rexlängert war, enlyindete sich der llufs sehr häufig; während dov knofeme schornstein strömo vou ontzïndetem fufse ausspje, zeigte die iufere Yemperatur des gemautarten Schornsteins, dak es dorin uirhi branate. Wenige Tage nach einer reichlichen Aussprühung roa Funken, fand man das Manerwerk vollkammen reiu, walirend sich an den ezkalteraden Wanden des Kupfers schon bine 1 . Cenlimeter dicke Lage von Rufa abgesetzt hatte. Scharnsteine pan Eisenblech zeigen ganz abnliche krscheinungen. 
Die Kohle wirkt hier gewissermafsen wie Platinschwamm In einam Gemenge ron Wasserstoffgas and Lafi ader wie durch Wasserstofigas reducirtes Eisenoxyd, dax pyrophorisob jst weun nitht eine suhr hahe Tenperatur seine Porozität zerstört hat. Mă erhält einen deutlichen Beweis ron der pyrophorischen Eigenschaft des Rulses, wenn man diese Substanz in einem rerschlossenen Tiegel glüht und den Deckel vor dcm valliggen lirkolten wognimmt. In wenigen Augenblicken geräth die Obertiäche des Ifufses ins Giülen durch fraiwillige Temperuturerhöhung. Dieso Erscheinungen ereignen sich of bei der Fabrikatinn des Beinschwarzes; der Sauerstoff wird a leicht durch die Kohle condensiri, dafs die Knochen weifs werden, wenn sie nur vor dem villigen Erkalten dec Laft ausgeselzt werden. *)

In Schiefspulyerfabriken ereignete es sich schon. dafs pulrerisirte Molzhohle freiwillig Feuer ing durch A bsorption ron Luft bei gewöhnlicher 'Temperatur.

Der bei der Zersetzung der in der Steinkohle enthaltenen Schwefelkiese entstehente Schwefelwasserstoff vermchrt vieileicht die pyrnphorische Eigenschaft des Rufses; jedermann wcifs, , nit welcher Leichtigkeit poröse Kohlen dieses Gas ubsorbiren. Nach Th. v. Sausure absorbirt Holghohle ihr

-) Die grob pulverisirte thierische Kohle zeigt noch eine undere Btgenschaft, weiche wit der eben angefulirtes einige Analugie hat; jämlich das Vermögeu unter Beihulfe einer höhereu Turuperatur dus Wasser zu zersetzen. Wenn diese frisch bereitele Kohle wit etwas Wasser befeuchtet wird, so steigt bald die Temperatpr und das Beinschwarz verliert einen Theil seiner Koble und wird grea.

Die uemliche Beobachtuug ist für die körnige Kohle anwendbar, welche zur Zuckerfabrikation gedient hat, nur kauu iu diesem Fall die Temperalurerhöhung zun Thell Jer Gäliruug des 2achers zu. geschrieben werdeu, wonit die Kohle impraguirt ist. Diese Temperaliırerhöbung ist no beträchllich bei grofien Massea, und die Verbrenung der Kohle macht solche Fortschritte, daf der Rück. stand völlig weifs wird, und bald our nus phosphorsaurem und hoh. lensaurem Kalk besteht. 
55facheg Volum unl condensirt melur ale das 9fache Volum Sanersioff. Etenso fand braconnot bei der Analyse von, allem Auschein nach won Steinkohle herrührendem, Rulse 3,30 Proc. schwefelsaures Ammoniak, welches am grofsen Theil anr durch Wirhung des Yufi suf das durth dic Kohle absorbirte sthwefelwaske:suofi-A numoniak entstanden seyn kann.

Wenn dis kntrindungsfalle in gremanerten Schornsteinen seltener sis in metalleyen sind, so ruhrt diefs daher, weil das gleichtormiger erhitye Mauerwerk den Absatz des Rulsen nicht so crleichtert, unj weil die abgesetzte hohle sich nicht in für die Absmption das cases so giunstigen Umständen beAndet. In eisernen oder knpfernen Rölren wird diese $\mathbf{A b}$ sorption, hauptsïchlich wems der Ifeerd nicht geepeist wird, erleichtert durch die Rerïlıung des beständig durch die äussere Luft, erkilteien Metalls.

Es bleibt nor:h die Frnge zu entucheiden übrig, ob nämlish dos Sichwefalk upler durch seine Umwandiung in Sulphat anch eilie bediugende Lisactie der Entzïndun: in den kupfernen Röhren seyn kann. Das Eisen ist wie das Kupfer in Stande Schwefolwasserstofi au zersetzea, selbat bel yewohnlicher Temperatur; das Scliwefeleisen kann dieselben Reactionen wie das Saliwefelk opfer remanasen; die freiwillige Hatzündung der Naunschicter in den Alannwerken ist ein Beweis davon. Fs gibr liäufige Beispiele dir freiwilligen Fintä̈ndung von Schwefulkiesreichen Steinkuhlew; diese Entzündungen finden manchmal selbat in den Minen atat und die Verbrennung betindet sich in diesem letzteren Falle gevifs in viel weniger günstigen Umatänden sls in den wetallenen Rohren, wo ein sehr fein zertheiltes Sulfür in Berihrong mit gleichtalls wehr fein zertheilter Kohle iet, die bis zur Sättigung den Sauerstoff dev Luft condensiren und so die Umwandlung des Sulfürs in Sulphat erleichtern kann. Ws bestäude demnach ein gewisser Zusammenhang zwischen der absorbirenden und pyrophoxi- 
chen Wirknng der Kohle und der Erzeugung der Sulphate in den metallenen Röhren und diese beiden Fnizändungaurachen konnten vehr Jeicht gleichzeitig wirken. Die schnelle Verderbnifs, welcher die eisernen Schornsteine unterliegen, scheint die Bildung des fraglichen Sulfürs zu bestätigen, woron übrigens der Beweis durch die Analyse der herabgefalleaen Asche crlangt wurue. Diese Asshe enthält sehr viel schwefalsaures Bisenoxydul und Schuppen von Sulfur, das zum 'Theil in Sulphat amgewandelt ist.

Wenn die Awwendung wetallener Schoristeine in einigen seltenen Fällen zulässigs seyn kann, so reichen doch wenige Worte hin, um zu zeigen, wie venig ökonomisch sie sind. Aufser dem grofien Verlisat an Wärme, der durch die metallenen Röhren eutsteht und der den Zug vermindert, haben diese Röhren nocb deu rachtheil einer anfserordentlich schnellen Veränderung. Fin kupfernes Hohr ron 67 Kilogr. uroprünglichem Gewicht vertor in dem Zeitraume von swei Jallren 17 Kilogr. Meiall and war, beonders an der Naht sthon durchfressen, währead an der äufseren Oberfläche keine merkliche Veränderung statt fand. Der Verlust betrag im Jahr anf den Quadrat-Mcter Oberfiche 2 Kiiogr. 003 Gr. Die Heftigkeit dieser Zerstörung muls immer von der Mächtigkeit des Heerdes, dem Durchmesser der Röhron, der mehr oder weniger schwefligen Besrhaffenheit der Stcinkohle abhïngen, und sie ist wahrscheinlich nicht für jede Höh der Möhren gleich. So verior eine Zöhre von Kupfer von Y Yet. 50 Centimeter Lange, und einem Durchmesser ron $0, i \gamma$, die ala Schornstein in einer Zuckerraftiverie diente, in Zeit von 2 Jahren nur $23 \frac{9}{2}$ Kilogr. an Gewicht, was im Jahr auf deu QuadratMeter nur 1 Kil. 0,61 Gr. beträgt.

Diece Arbeit fübrie zu folgenden Schlüssen, die sowohl für die Gewerbe als auch für die iffentliche Sicherheit und Gesundheit von lebhaftem Interesse sincl. 
1. Die Schumsteine ron Kupfer erieiden bei fluem Gebrauche, wonn die Hecrde mit Steinkohlen gespoist werien, cine schnello Zirstörung: es bildet sich an den inneren wän. den eine Krote rain Sehwefalkupfer, das nach und nach in *nsserfreies Sulphat ibhergcht, dos in dicsem zustande darch. den Luftrag uarh oben gerissen wird und auf die umliegenden Datcher zurickfält. Das Hegenwasper !ist dizsits Salz anf und fuhrt es in die als Rescruir dienemien Cistermen. Das Was. ser der Cisternen kann wechselnde Quandiäten je wach der Lage ater Dichex, des Richtung des Windes und besnnuers mach nach der Capacitä der Cisternen ron diesern gifigen Salze entialten.

2. Die angeführte \%orstörnng gill auch für eiserue Wohren: es lildet sich, unter gleichen Umständen Schwefeleisen und scliwefeloames Eisenoxydul io bedeutender Menge.

*. bie Anwendang der metallenen Röhren als Sehnrusteine veranlafot Entzïndungen, deren Iläufigkeit der pyrophasischun Figenschaft des llufses und vielleicht auch der Imwandlung des Schweitimetalls in Sulphat zugeschrieben werden mufo.

*. Die Zerstinung dieser Schornsteine geht so rasch rop sieh und dir dadurh vermeathte fitfahr ist so bedeutend, dais es himeichen wird. die iu dieser Notiz bezpichnpien Thatsactie's wrulegur, um dea fewerbsmann von einem 6 brawdh abzisingeses, der aufser den peruniären Nachtheil, seine llube and dic dor Nachborn antergräbt. 